\title{
Global Oscillations of Masing Disks in Megamasers
}

\author{
Atsuo T. Okazaki \\ Faculty of Engineering, Hokkai-Gakuen University, Toyohira-ku, \\ Sapporo 062-8605, Japan
}

\begin{abstract}
We study the characteristics of global oscillation modes of masing disks in megamasers and the effect of the modes on the disk kinematics. We find that the eccentric mode is responsible for the observed sub-Keplerian velocity distribution of the maser source of NGC 1068, whereas in the masing disk of NGC 4258 the warping mode is dominant so that the angular rotation velocity remains near Keplerian.
\end{abstract}

\section{Introduction}

Recently, VLBI observations have revealed the detailed spatial and velocity distribution of maser emission in megamaser sources. In this paper, we studied the effect of global oscillation modes on the kinematics of masing disks in megamasers, using a simplified disk model.

\section{Eccentric Mode in the Masing Disk of NGC 1068}

Greenhill et al. (1996) found that the line-of-sight velocity of the redshifted maser sources in the nucleus of NGC 1068 decreases as $r^{-0.31}$. This sub-Keplerian line-of-sight velocity distribution can be attributed to the fundamental eccentric mode shown in Figure 1.

\section{Warping Mode in the Masing Disk of NGC 4258}

The position and velocity distribution of the water megamaser sources at the center of NGC 4258 are fitted well with a warped Keplerian disk model (Miyoshi et al. 1995). We found that the characteristics of the fundamental warping mode shown in Figure 2 agree well with the observed Keplerian velocity distribution of maser sources of NGC 4258.

\section{References}

Miyoshi, M., Moran, J., Herrnstein, J., Greenhill, L., Nakai, N., Diamond, P., and Inoue, M. 1995, Nat, 373, 127

Greenhill, L. J., Gwinn, C. R., Antonucci, R., and Barvainis, R. 1996, Ap.J., , 472, L21 

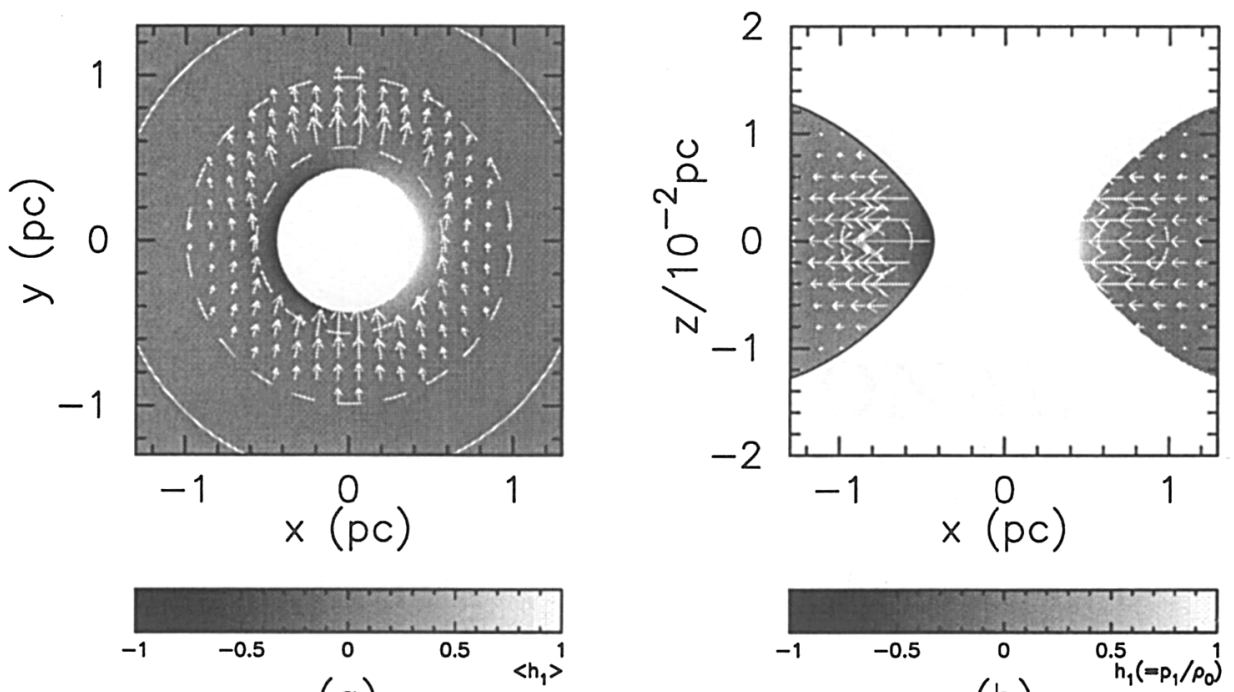

(b)

Figure 1. Fundamental eccentric mode in the masing disk of NGC 1068. (a) The $(r, \phi)$-distribution of the perturbations averaged vertically over the upper half of the disk. (b) The $(r, z)$-distribution of the perturbations. A gray-scale representation denotes the enthalpy perturbation $h_{1}$. Arrows superposed on the gray-scale plot are the perturbed velocity vectors in the maser emission region (surrounded by dashed lines).
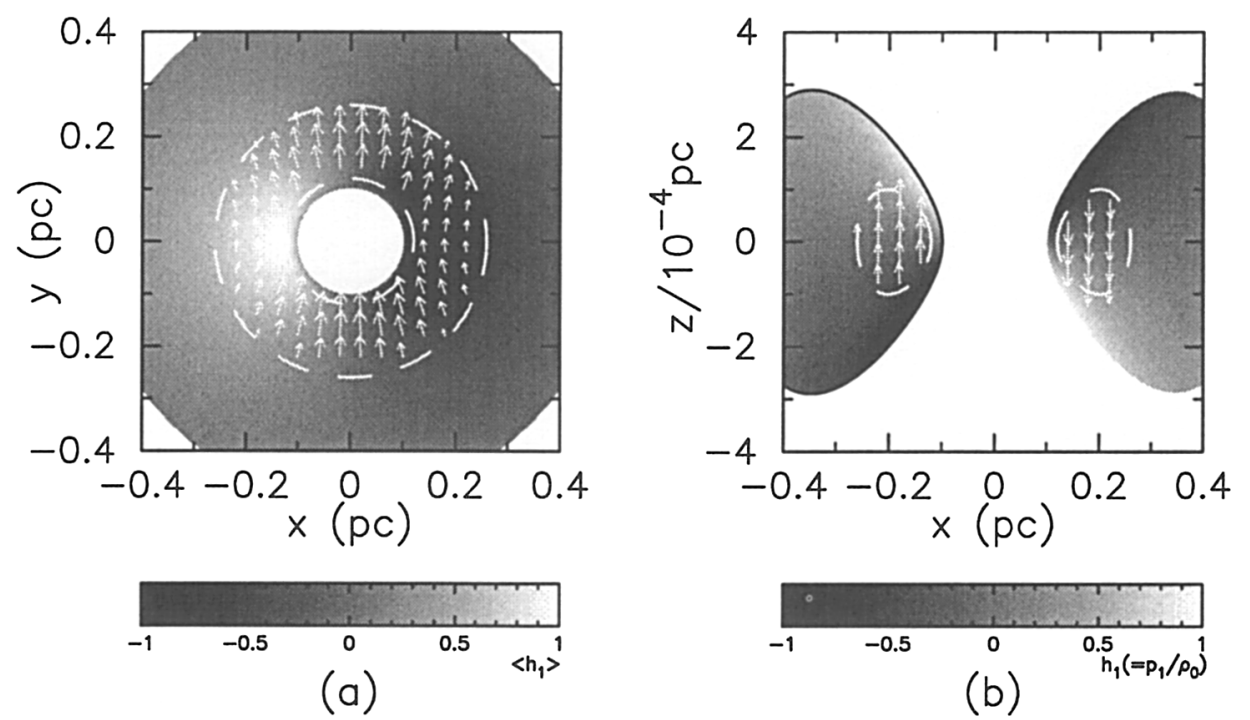

(b)

Figure 2. Fundamental warping mode in the masing disk of NGC 4258. The format of the figure is the same as that of Figure 1. 